\title{
Comorbidities, mortality and causes of death among patients with tuberculosis in Denmark 1998-2010: a nationwide, register-based case-control study
}

\author{
Andreas Fløe, ${ }^{1}$ Ole Hilberg, ${ }^{2}$ Christian Wejse, ${ }^{3,4}$ Rikke Ibsen, ${ }^{5}$ Anders Løkke ${ }^{1}$
}

\begin{abstract}
- Additional material is published online only. To view please visit the journal online (http://dx.doi.org/10.1136/ thoraxjnl-2016-209240)

${ }^{1}$ Department of Respiratory Diseases, Aarhus University Hospital, Aarhus, Denmark 2Department of Respiratory Diseases, Sygehus Lillebaelt, Vejle, Denmark

Department of Infectious Diseases, Aarhus University Hospital, Aarhus, Denmark ${ }^{4}$ Department of Public Health, Center for Global Health, Aarhus University, Aarhus, Denmark 5i2minds, Aarhus, Denmark
\end{abstract}

\section{Correspondence to} Dr Andreas Fløe, Department of Respiratory Diseases, Aarhus University Hospital, Nørrebrogade 44, Aarhus DK8000, Denmark; andrniel@ rm.dk

Received 26 July 2016 Revised 19 May 2017 Accepted 10 July 2017 Published Online First 4 August 2017

\section{ABSTRACT}

Objective To evaluate the impact of comorbidities, age and clinical presentation of TB on mortality among Danish patients with TB.

Methods Danish patients with an ICD-10 (International Statistical Classification of Diseases and Related Health Problems 10th Revision) diagnosis of TB in 1998-2010 were identified in the National Patient Registry and matched with controls (1:4) on age, gender, civil status and geography. Comorbid diagnoses up to 3 years before and after TB diagnosis or enrolment as control as well as survival data were obtained from national databases

Results We included 8433 cases and 33707 controls. Respiratory diseases were the most common comorbidities among cases $(12.4 \%$ of cases, $3.8 \%$ of controls $(p<0.001))$. Overall HR of death was $2.45(2.31$; 2.59). Relative mortality was especially increased among younger adults (HR 8.70 (95\% Cl 5.53 to 13.69) among the 30 to 39 -year-olds). While overall mortality increased with Deyo-Charlson comorbidity (DCC) score, relative mortality among cases was highest in the low-DCC group. Additionally, male gender, low income and central nervous system TB were risk factors for death among TB cases. The most common cause of death in both groups was non-lung cancers, among TB cases followed by COPD, TB and lung cancer, all being significantly more common among TB cases.

Conclusion In Denmark, TB carries substantial mortality. Among those who die, $12 \%$ are reported to die from TB. A high relative mortality among younger adults underscores the importance of continually targeting high-risk TB groups in low-incidence countries.

\section{INTRODUCTION}

TB is generally considered a major health problem in developing countries where the disease continues to cause significant morbidity and mortality. ${ }^{1}$ Despite a remarkable drop in TB incidence over the past century in Western Europe, TB remains a considerable health issue among foreign-born residents and recent immigrants from endemic countries, ${ }^{2}$ as well as in certain groups of socially marginalised citizens. ${ }^{34}$ In addition to the well-described correlation between HIV infection and $\mathrm{TB},{ }^{5}$ previous studies have documented a correlation between TB and diabetes, ${ }^{67}$ alcohol abuse, ${ }^{8}$ smoking ${ }^{10}$ and COPD. ${ }^{11}$ Previously, an association between TB and lung cancer has also been reported, ${ }^{12}$ although it may to a large extent

\section{Key questions}

What is the key question?

- Which patients with TB are at highest risk of dying during and after their disease, and what do they die from?

What is the bottom line?

- To explore actual causes of death and comorbidity patterns among patients with TB in a low-incidence setting.

Why read on?

- While absolute mortality increases with age, younger patients with TB and those with few comorbidities are at high risk relative to matched controls.

represent shared risk factors. In Western Europe, these coexisting diseases and conditions may be important drivers of TB for several reasons: immune suppression due to comorbid conditions may increase the risk of reactivation of latent $\mathrm{TB}$ infection (LTBI) and may increase the risk of TB development directly following infection. Importantly, most of these conditions are over-represented among socially marginalised citizens which exacerbates the effect of the increased risk of contracting Mycobacterium tuberculosis (MTB). Of the estimated 1.5 million TB-related deaths in $2014,{ }^{1}$ the majority occurred in low-income countries. Nevertheless, a strikingly high all-cause mortality is seen among patients with $\mathrm{TB}$, also in Western Europe, ${ }^{2}$ with substantial socioeconomic inequality in the mortality risk in most European countries. ${ }^{13}$ In order to improve TB outcomes in Western Europe by targeting the most relevant risk groups and to reduce mortality among our patients with $\mathrm{TB}$, we need a better understanding of the actual comorbidity patterns in $\mathrm{TB}$ and their impact on survival, as well as of the actual causes of death among patients with TB. We therefore aimed to describe comorbidity-related mortality and causes of death through a 12 -year period in a complete national TB cohort.

\section{METHODS}

In Denmark, all hospital contacts are registered in the National Patient Registry (NPR). The NPR holds administrative information, primary and secondary 
diagnoses and information on diagnostic and treatment procedures using the International Statistical Classification of Diseases and Related Health Problems 10th Revision (ICD-10). Primary sector contacts (general practice and specialist care) are recorded in the National Health Security Registry. We extracted data on all patients with a primary or secondary ICD-10 diagnosis of A15 through A19 in the period 1998-2010 (12 years). This includes pulmonary TB (PTB) and extrapulmonary TB (EPTB), but not latent TB (LTBI). Data were available for the entire observation period, allowing us to trace patients both retrospectively and prospectively relative to the time of diagnosis, as described elsewhere. ${ }^{14}$

The Danish Civil Registration System contains information about cohabitation status, marital status, education, income, pensions, and so on, ${ }^{15}$ but it does not contain information on ethnicity, according to Danish law. We randomly selected controls matched by age, gender, geographical area and marital status, but we refrained from matching on income status or education level, as this would compromise the ability to find matching controls in a close geographical area, and would limit the possibility to study the effect of such socioeconomic determinants. The patient-to-control ratio was 1:4. If a patient or control was not present in the registry on 1 January in a given year because of death, imprisonment or emigration, the corresponding control or case was excluded.

Information pertaining to the patient before and after his or her TB diagnosis was extracted from the databases for the years 1998-2010. Comorbidity data prediagnosis were obtained in the 3 years preceding the TB diagnosis; therefore, prediagnosis comorbidity and comorbidity-related mortality could be evaluated for the years 2001-2010. Equally, postdiagnosis comorbidity could be evaluated for the years 1998-2007. Morbidity data were extracted as primary and secondary diagnoses and further classified into main disease groups in accordance with the WHO ICD-10 criteria. Diagnostic codes were grouped manually and reported on the basis of their statistical significance and relevance. From comorbidity data, we calculated Deyo-Charlson comorbidity (DCC) score. The DCC includes 17 diagnostic groups in a weighted score that associates with 1 -year mortality, in a model adapted for use with administrative register data, ${ }^{16}$ and for use with ICD-10 diagnoses. ${ }^{17}$

Data collection and manuscript preparation were completed in accordance with the STROBE (STrengthening the Reporting of OBservational studies in Epidemiology) statement. ${ }^{18}$

\section{Statistical analysis and ethical considerations}

The study was approved by the Danish Data Protection Agency. Ethical approval was not required as data handling did not involve revealing the identity of any patients or control subjects. Comorbidity data were analysed using a conditional logistic regression model, yielding ORs with 95\% CIs. Data reported as percentages were compared using Pearson's $\chi^{2}$ test. Survival data were reported using the Kaplan-Meier survival function. Relative survival was expressed as HRs derived from a Cox proportional hazards model. Statistical analysis was performed using SAS V.9.1.3. A significance level of 0.05 was assumed for all tests.

\section{RESULTS \\ Study cohort}

We extracted survival data on 8433 TB cases and 33707 matched controls (figure 1). Comorbid diagnoses in the 3 years preceding TB diagnosis were captured from 6713 cases and
28217 matched controls. Hereof 3750 (55.9\%) of cases and $15681(55.6 \%)$ of controls were male, and 32.9\% were 30-49 years old. Postdiagnosis comorbidities could be retrieved from 7311 cases and 31194 controls (1998-2007). The total cohort and the subgroups with pre and postdiagnosis comorbidity information were comparable with regard to age, gender and educational level (data not shown).

\section{Comorbidities}

At the time of TB diagnosis, the majority of TB cases $(86.34 \%)$ had a DCC score of 0 , although the corresponding number for controls was significantly higher $(93.99 \%)(p<0.001)$. Respiratory diseases, including COPD, were significantly more common among cases than among controls before TB diagnosis $(12.4 \%$ of cases vs $3.8 \%$ of controls; OR 2.28 (95\% CI 2.08 to 2.51$)$ ) as well as after diagnosis $(15.3 \%$ of cases vs $4.3 \%$ of controls; OR 2.33 (95\% CI 2.11 to 2.56)). Other disease categories being significantly more common with cases than controls in the 3 years preceding the TB diagnosis included haematological disorders $(2.1 \%$ of cases vs $0.4 \%$ of controls, OR 2.20 (95\% CI 1.72 to 2.80$)$ ), mental disorders (4.8\% of cases vs $1.4 \%$ of controls; OR 1.87 (95\% CI 1.52 to 2.12$)$ ), non-TB infectious diseases $(4.9 \%$ of cases vs $1.5 \%$ of controls; OR 1.78 (95\% CI 1.53 to 2.06)), dermatological diseases $(4.8 \%$ of cases vs $1.8 \%$ of controls; OR $1.66(95 \%$ CI 1.43 to 1.91$)$ ) and gastrointestinal disorders $(11.3 \%$ of cases vs $5.1 \%$ of controls; OR 1.44 (95\% CI 1.31 to 1.59 )). Neoplasms and cardiac disorders were both more common among cases than among controls, but not statistically significant. Online supplementary 1 provides a complete overview of pre- and postdiagnosis comorbidities grouped into main ICD-10 categories and by single disease category.

\section{Mortality by age and gender}

Overall mortality in the total cohort is illustrated in figure 2 . The HR for death was $2.45(2.31 ; 2.59)$, primarily reflecting elevated mortality in the first 2 years after the TB diagnosis.

Death was a rare occasion in subjects under 20 years of age, among cases as well as controls; and no significant difference was observed in mortality in this age group. For all age groups above 20 years of age, mortality was significantly higher among cases than among controls (table 1 and online supplementary 2). While absolute mortality increased with age, relative mortality was highest among younger adults from 30 to 39 years of age, as depicted by an HR of $8.70(5.53 ; 13.69)$ (table 1). Mortality was higher among men than among women, as depicted by an HR for men of $3.58(3.27 ; 3.92)$ and $3.01(2.69 ; 3.36)$ for women.

\section{Impact of DCC score, TB location and income on mortality}

Of the $6713 \mathrm{~TB}$ cases and 28217 controls providing prediagnosis information, $1512(22.5 \%)$ cases and 2581 (9.1\%) controls passed away. The mortality pattern was influenced by prediagnosis comorbidity burden and by localisation of TB (figure 3). Mortality was significantly higher among cases than among controls when the DCC was 0 (Kaplan-Meier survival estimate at 5 years: $0.85(0.84 ; 0.86)$ vs $0.95(0.948 ; 0.953))$ and score $1-2(0.55(0.49 ; 0.60)$ vs $0.67(0.63 ; 0.70))$. With DCC score of 3 or more, an insignificant trend of inferior survival in the control groups was seen (Kaplan-Meier survival estimate at 5 years: $0.40(0.24 ; 0.57)$ vs $0.35(0.21 ; 0.48))$. Importantly, though, the groups with DCC score of 3 or more included few subjects; $114(1.7 \%)$ cases and $115(0.4 \%)$ 


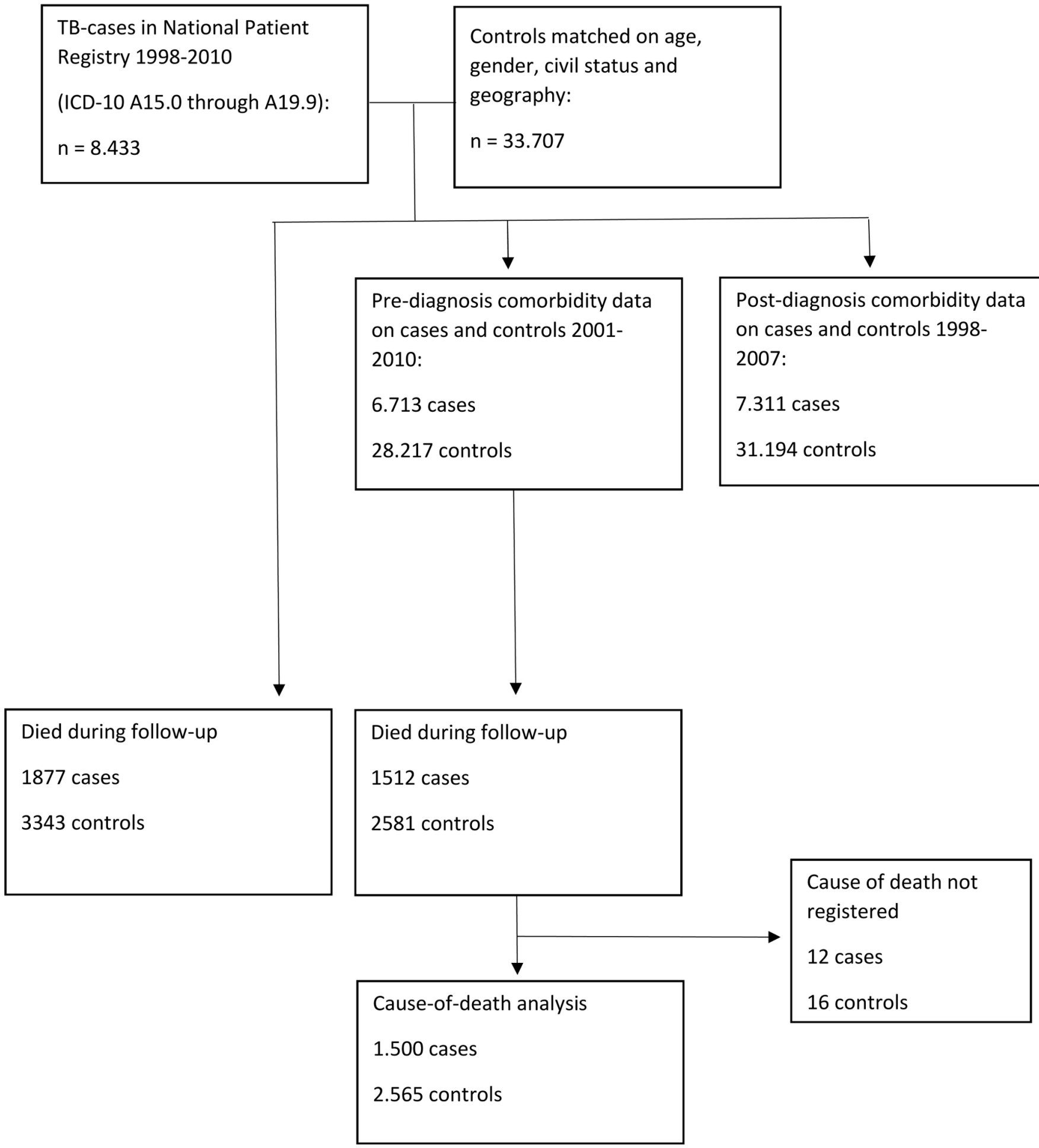

Figure 1 Inclusion of patients with TB and matched controls for survival and comorbidity analyses. ICD-10, International Statistical Classification of Diseases and Related Health Problems 10th Revision.

controls. Compared with controls, mortality was significantly increased for all forms of TB (figure 3 and table 2). Adjusting for DCC score did not change this correlation significantly; DCC-adjusted HR was $3.36(3.11 ; 3.64)$ for PTB, 1.84 (1.48; $2.30)$ for EPTB and $15.30(6.34 ; 36.93)$ for central nervous system (CNS) TB. A Cox proportional hazards analysis performed only on TB cases confirmed that CNS TB carried significantly elevated mortality, while EPTB carried significantly lower mortality than PTB (table 2). Additionally, this analysis confirmed DCC score, age and male gender as risk factors for mortality, while increasing income was associated with reduced mortality.

\section{Cause of death}

Information on cause of death was available for 1500 cases and 2565 controls who died during follow-up. Non-pulmonary cancer was the leading cause of death in both groups, although 


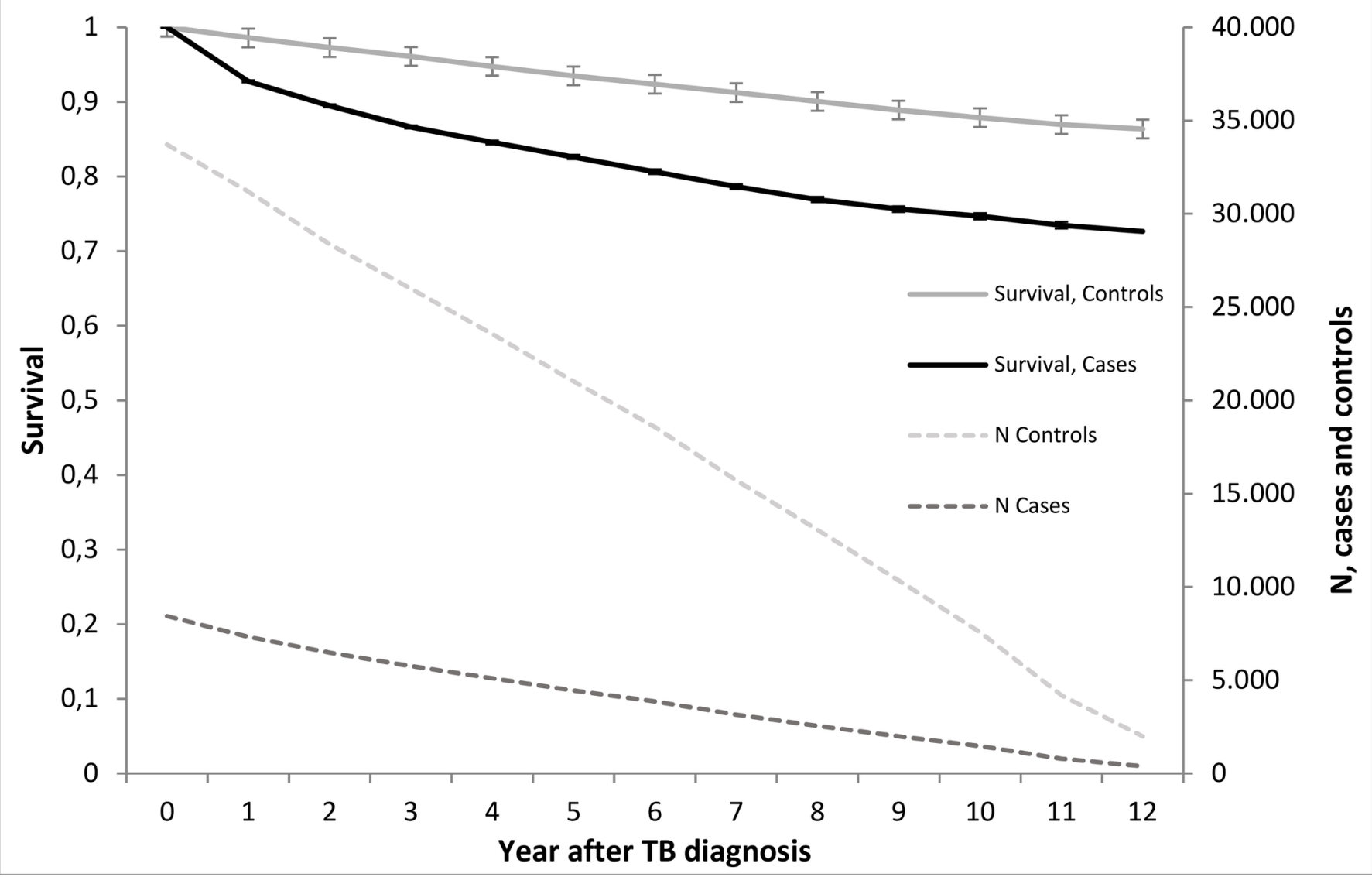

Figure 2 Kaplan-Meier survival curve (left vertical axis) and number of TB cases and matched controls (right vertical axis) up to 12 years after TB diagnosis or enrolment as controls. Case: TB case with an ICD-10 (International Statistical Classification of Diseases and Related Health Problems 10th Revision) diagnosis in the spectrum A15 through A19 in 1998-2010. Control: Matched controls, matched on age, gender, civil status and geography.

it was significantly more common among controls than among cases (table 3). Among TB cases, non-pulmonary cancers were followed by COPD (including exacerbations), TB and lung cancer as most common causes of death. COPD, TB and lung cancer were all significantly more common causes of death among TB cases than among controls.

\section{DISCUSSION}

We have here presented the findings from a national, retrospective, register-based study reporting on comorbidities, survival and causes of death among Danish patients with TB and matched controls. We found that comorbidities were more common among patients with TB than among matched controls, before as well as after the TB diagnosis. The most frequently reported comorbidities were respiratory diseases, which were significantly more common among patients with $\mathrm{TB}$ than among matched controls. Overall survival was inferior for TB cases compared with matched controls. Absolute mortality increased with increasing number of comorbidities and with increasing age; but high relative differences were observed for patients with a low comorbid burden as well as for younger adults. Non-pulmonary cancers were the most common causes of death among patients with TB as well as controls. These were followed by COPD, TB and lung cancer as most common causes of death among patients with $\mathrm{TB}$, and by stroke, heart disease and lung cancer among controls.
The higher frequency of respiratory diseases among patients with TB in the 3 years preceding the TB diagnosis needs some consideration. Respiratory diseases included COPD and asthma, but also pneumonia; in some cases the latter may be the initial presentation of what later turns out to be PTB. Nevertheless, as can be calculated from online supplementary 1 , respiratory diseases remained substantially more common among TB cases than among controls after exclusion of pneumonia diagnoses. To a large extent, this probably reflects shared risk factors, most importantly tobacco smoking. Smoking significantly increases the risk of MTB infection as well as the risk of active TB. ${ }^{19}$ Impaired ability of alveolar macrophages to control intracellular MTB growth in smokers may partly explain this finding. ${ }^{20}$ Furthermore, increasing evidence supports that COPD is a risk factor for TB beyond what is explained by smoking patterns ${ }^{2122}$; hence, several studies find that the risk of TB is especially elevated in patients receiving high-dose inhaled corticosteroids. $^{22}{ }^{23}$ However, careful attention should be paid to the fact that high-dose steroids are primarily dispensed to those with severe COPD and, also, prescription of steroids may in some cases be commenced on basis of increasing pulmonary symptoms that would later turn out to be caused by co-occurring TB.

Other disease groups that were seen more commonly among patients with TB included gastrointestinal diseases, skin diseases and, to a lesser extent and non-significantly, malignancies. Several 
Table 1 Share of cases and controls who died during follow-up by age and gender groups and by clinical presentation of TB, and corresponding hazard rates of mortality

\begin{tabular}{|c|c|c|c|c|c|c|}
\hline & \multicolumn{2}{|l|}{ Case } & \multicolumn{2}{|l|}{ Control } & \multirow[b]{2}{*}{ HR } & \multirow[b]{2}{*}{$95 \% \mathrm{Cl}$} \\
\hline & $\mathrm{N}$ & Share dead (\%) & $\mathrm{N}$ & Share dead (\%) & & \\
\hline \multicolumn{7}{|l|}{ Age group (years) } \\
\hline $0-9$ & 374 & 0.5 & 1609 & 0.2 & 2.84 & $(0.47 ; 17.03)$ \\
\hline $10-19$ & 459 & 0.7 & 2116 & 0.2 & 4.09 & $(0.83 ; 20.30)$ \\
\hline $20-29$ & 786 & 1.7 & 3474 & 0.3 & 7.51 & $(2.98 ; 18.88)$ \\
\hline $30-39$ & 1137 & 5.1 & 4883 & 0.6 & 8.70 & $(5.53 ; 13.69)$ \\
\hline $40-49$ & 1139 & 14.0 & 4747 & 2.8 & 5.85 & $(4.60 ; 7.44)$ \\
\hline $50-59$ & 944 & 27.6 & 3813 & 6.1 & 5.68 & $(4.71 ; 6.85)$ \\
\hline $60-69$ & 737 & 40.8 & 3010 & 12.5 & 4.34 & $(3.68 ; 5.11)$ \\
\hline $70-79$ & 723 & 56.0 & 2911 & 30.0 & 2.61 & $(2.29 ; 2.96)$ \\
\hline $80+$ & 414 & 74.6 & 1654 & 55.6 & 2.00 & $(1.73 ; 2.31)$ \\
\hline \multicolumn{7}{|l|}{ Gender } \\
\hline Male & 3750 & 24.8 & 15681 & 9.7 & 3.58 & $(3.27 ; 3.92)$ \\
\hline Female & 2963 & 19.6 & 12536 & 8.4 & 3.01 & $(2.69 ; 3.36)$ \\
\hline \multicolumn{7}{|l|}{ TB localisation } \\
\hline Pulmonary TB & 5336 & 23.7 & 21136 & 9.7 & 3.55 & $(3.28 ; 3.83)$ \\
\hline Extrapulmonary TB & 1064 & 12.7 & 4219 & 7.5 & 2.01 & $(1.62 ; 2.49)$ \\
\hline Central nervous system TB & 106 & 26.4 & 421 & 2.9 & 15.76 & $(6.88 ; 36.09)$ \\
\hline All (N) & 6713 & & 28217 & & & \\
\hline
\end{tabular}

mechanisms may explain these findings, and the causality is not always obvious as TB may give rise to symptoms several months before it is diagnosed. ${ }^{24}$ All the above-mentioned ICD-10 diagnosis groups may lead to immune suppression, either by the disease mechanism per se or because of the use of immunosuppressive treatment, including corticosteroids. ${ }^{25}$ The diagnosis groups may, however, also represent shared risk factors as is also the case with regard to respiratory diseases; for example, smoking increases the risk of many non-pulmonary cancers as well as diseases like rheumatoid arthritis. ${ }^{26}$ Endocrine diseases, mainly diabetes mellitus, were also more common among TB cases than among controls. Diabetes is a well-known risk factor for active $\mathrm{TB}^{27}$ as well as for reactivation of LTBI. ${ }^{28}$ There may also be a confounding effect of higher diabetes risk among socially marginalised citizens who are also at risk of TB. ${ }^{29}$ The same pattern applies to alcohol abuse, which implies an elevated risk of $\mathrm{TB},{ }^{30}$ which is also likely an effect of a compromised adaptive immune response. ${ }^{31}$ Although studies in mice support a real, increased risk of progression to active TB with increased alcohol consumption, ${ }^{32}$ alcohol abuse is also tightly related to social marginalisation and population groups with a high incidence of TB. In the ICD-10 classification system, alcohol abuse is grouped under mental disorders. In our study, mental disorders were also significantly over-represented among TB cases, and alcohol abuse constituted the most prevalent single diagnosis in this group. Alcohol abuse, however, may also affect other disease categories. For example, the elevated prevalence of gastrointestinal diseases may reflect liver disease due to alcohol abuse.

Overall mortality was increased among patients with TB, illustrated by an HR of 2.45. This is in accordance with previous studies from high-income countries ${ }^{33} 34$ which have also found that risk factors for mortality include non-infective comorbidities, sputum smear positivity and alcohol or substance abuse. ${ }^{34}$ In the present study, the comorbidity burden, as assessed by the DCC score, significantly impacted mortality. With a low comorbidity burden, overall mortality was low, but significantly higher among TB cases than among controls. This calls for consideration; it may to some extent represent mortality from TB, primarily among socially marginalised citizens who may present late for treatment. In the group with a high DCC score, overall survival was poor, and not significantly different between cases and controls. This group probably included a number of patients with malignant diseases or other life-threatening disease who may develop TB due to compromised immunity. Also, the thorough diagnostic procedures applied to patients in whom cancers are suspected may increase the detection rate of $\mathrm{TB}$ in late disease stages, which would skew TB cases towards the more seriously ill patient categories. When applying the DCC, it should be noted that HIV infection per se does not yield a high score, while AIDS yields a high DCC score. As TB is an AIDS-defining disease, any HIV-infected patient with TB would end up with a high DCC score. Though this is a serious situation, such patients may have a better prognosis than patients with a high score due to malignant diseases, and so on, and this may contribute to the trend towards better survival among patients with $\mathrm{TB}$ in this group. On the other hand, HIV TB is a rare manifestation in Denmark, with a median of nine reported cases per year during the study period. ${ }^{35}$

We observed a strikingly impaired survival among TB cases under 40 years of age; and when considering TB cases alone, increasing income was significantly associated with improved survival. It is well known that $\mathrm{TB}$ is especially a concern among younger, socially marginalised citizens, among whom high degree of clustering is seen due to active transmission. ${ }^{36}$ Our findings suggest that also mortality is a serious concern in these groups, and it can be speculated that a large fraction of the $12 \%$ of deaths among TB cases, where TB was reported as the cause of death, happened in these groups. Further studies of the risk for directly TB-related death among different age groups and socioeconomic categories 
A
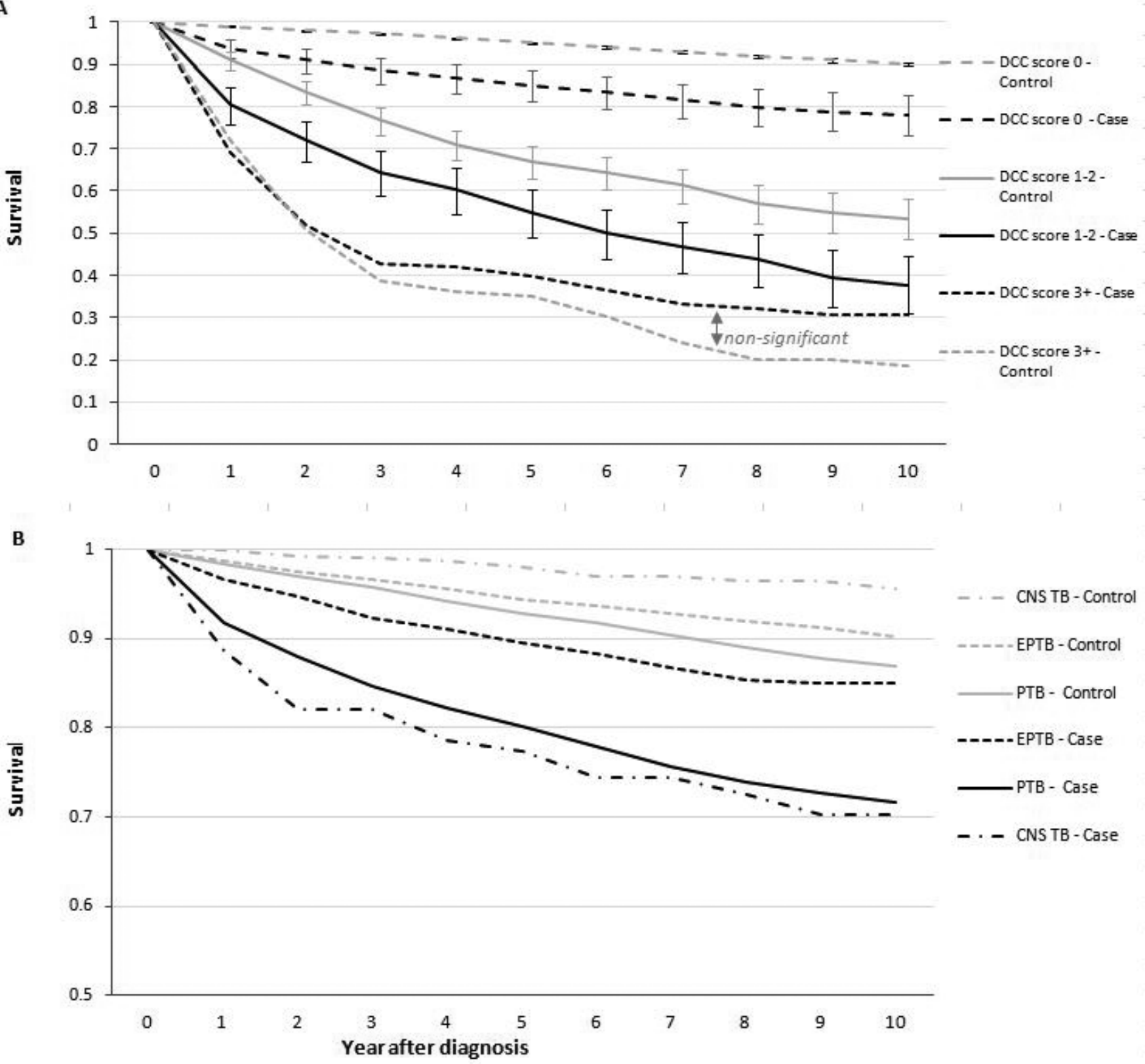

Figure 3 Kaplan-Meier survival curves for TB cases and matched controls, stratified by DCC score based on information in the 3 years preceding TB diagnosis or enrolment as control (A) and stratified by localisation of TB disease (B). Vertical bars represent 95\% Cls. In panel B, case-to-control differences were significant within all three disease groups. CNS, central nervous system; DCC, Deyo-Charlson comorbidity; EPTB, extrapulmonary TB; PTB, pulmonary TB.

would definitely improve our understanding of the risk of unfavourable outcomes in specific patient groups with TB, as would further studies of short and long-term mortality and corresponding causes of death.

In our study, CNS TB accounted for $1.6 \%$ of TB cases. In keeping with previous studies, ${ }^{37}$ CNS TB cases carried substantially higher mortality than PTB and EPTB, underscoring the severe nature of this disease. PTB carried higher mortality than EPTB, which may reflect an age effect, as well as a tendency towards higher risk of PTB among patients with TB in socially marginalised high-risk groups. ${ }^{36}$

Our study was register based and has limitations with regard to the data acquired. First, by extracting TB cases from registered diagnoses, we relied upon correct reporting of diagnoses from clinicians, as the registries do not contain clinical or microbiological information that could be used to validate the diagnoses. In the National TB Register (Statens Serum Institut, Copenhagen), 5594 TB cases were reported during the study period. ${ }^{35}$ This gap may be explained by false positive diagnoses in our cohort as well as by under-reporting in the national TB register. In comparable countries, previous studies have found under-reporting of TB in the range of 7\%-27\%. ${ }^{38}$ A previous study evaluated the NPR diagnoses of TB and found that the diagnoses had sufficient predictive value (positive predictive value), ${ }^{40}$ but in view of the gap demonstrated here, a study evaluating patient records for non-reported patients with a TB diagnosis could put a light on risk factors for under-reporting, as well as on specific mortality patterns in this group. Second, it may be argued that our data do not represent current trends as our study period ended in 2010. However, trends 
Table 2 Crude and adjusted HR for death among Danish patients with TB by localisation of TB disease. The adjusted HR included comorbidity (by Deyo-Charlson comorbidity score), gender, age and income as explanatory variables in a multiple Cox proportional hazards model

\begin{tabular}{|c|c|c|c|c|}
\hline & Crude HR & $95 \% \mathrm{Cl}$ & Adjusted HR & $95 \% \mathrm{Cl}$ \\
\hline \multicolumn{5}{|l|}{ Localisation of TB } \\
\hline Pulmonary TB & Ref & & Ref & \\
\hline Central nervous system TB & 1.19 & $(0.80 ; 1.76)$ & 1.78 & $(1.19 ; 2.66)$ \\
\hline Extrapulmonary TB & 0.53 & $(0.44 ; 0.64)$ & 0.59 & $(0.49 ; 0.72)$ \\
\hline Deyo-Charlson comorbidity index (per point increase) & 1.36 & $(1.31 ; 1.42)$ & 1.27 & $(1.21 ; 1.34)$ \\
\hline Male gender & 1.28 & $(1.14 ; 1.43)$ & 1.47 & $(1.31 ; 1.65)$ \\
\hline Age (per year increase) & 1.06 & $(1.06 ; 1.07)$ & 1.06 & $(1.06 ; 1.07)$ \\
\hline Income (per log increase) & 0.97 & $(0.93 ; 1.01)$ & 0.83 & $(0.79 ; 0.87)$ \\
\hline
\end{tabular}

in TB prevalence have not changed significantly in Denmark since $2010,{ }^{35}$ and we are therefore inclined to believe that the utilisation of a 12-year study period eliminates year-toyear fluctuations in TB patterns. Third, a common pitfall in case-control studies is the presence of cases among controls. With a TB incidence of 6.5 per 100 000/year in Denmark, we consider the risk of non-reported TB cases in the control group to be very low. Nevertheless, we did find a single patient in the control group who was reported to die from TB. From our register-based data, it is not possible to determine if this represented a non-reported TB case or a wrongly reported cause of death. Fourth, we matched cases and controls on age, gender, civil status and geography. It can be argued that matching on education, income or other socioeconomic factors would allow for a more precise estimation of TB-related risk factors; however, this would also refrain us from analysing income as a risk factor for death, and to discuss the importance of socioeconomic effects on TB-related mortality.

In conclusion, we demonstrate that overall survival of Danish patients with TB is severely inferior to that of matched controls. The relative risk of dying after a TB diagnosis is elevated in particular among younger adults and among those with a low comorbidity burden, while the absolute risk of death increases with age and comorbidity burden. The fact that the TB disease remains a common cause of death among Danish patients with TB underscores the importance of continuing focus on early case finding and treatment, even in low-burden, high-income countries.

\section{CONCLUSION}

Mortality is high among patients with TB, also among younger patients and those with a low comorbidity burden. TB itself is a common cause of death.

Table 3 Causes of death among cases and controls who died during follow-up

\begin{tabular}{|c|c|c|c|c|c|c|c|}
\hline \multirow[b]{2}{*}{ Group } & \multicolumn{3}{|c|}{ Case } & \multicolumn{3}{|c|}{ Control } & \multirow[b]{2}{*}{ p Value } \\
\hline & $\mathbf{n}$ & Share (\%) & Rank & $\mathbf{n}$ & Share (\%) & Rank & \\
\hline Cancer, others & 207 & 13.81 & 1 & 505 & 19.70 & 1 & $<0.001$ \\
\hline COPD & 202 & 13.48 & 2 & 130 & 5.07 & 6 & $<0.001$ \\
\hline TB & 175 & 11.67 & 3 & 1 & 0.04 & 19 & $<0.001$ \\
\hline Lung cancer & 165 & 11.01 & 4 & 146 & 5.69 & 5 & $<0.001$ \\
\hline Heart disease & 161 & 10.74 & 5 & 502 & 19.58 & 2 & $<0.001$ \\
\hline Others* & 141 & 9.41 & 6 & 353 & 13.77 & 3 & $<0.001$ \\
\hline Conditions related to alcohol abuse & 109 & 7.27 & 7 & 78 & 3.04 & 10 & $<0.001$ \\
\hline Stroke & 58 & 3.87 & 8 & 208 & 8.11 & 4 & $<0.001$ \\
\hline Found dead, unknown cause & 45 & 3.00 & 9 & 128 & 4.99 & 7 & 0.003 \\
\hline Pneumonia & 45 & 3.00 & 10 & 87 & 3.39 & 9 & 0.497 \\
\hline Other infections (non-TB) & 41 & 2.74 & 11 & 66 & 2.57 & 12 & 0.757 \\
\hline Diabetes mellitus & 34 & 2.27 & 12 & 55 & 2.15 & 13 & 0.796 \\
\hline Interstitial lung diseases & 32 & 2.13 & 13 & 16 & 0.62 & 18 & $<0.001$ \\
\hline Abdominal disorders & 22 & 1.47 & 14 & 71 & 2.77 & 11 & 0.007 \\
\hline Dementia & 14 & 0.93 & 15 & 88 & 3.43 & 8 & $<0.001$ \\
\hline Renal insufficiency & 14 & 0.93 & 16 & 29 & 1.13 & 15 & 0.554 \\
\hline Liver diseases, non-alcohol related & 11 & 0.73 & 17 & 25 & 0.98 & 16 & 0.429 \\
\hline Haematological diseases & 9 & 0.60 & 18 & 21 & 0.82 & 17 & 0.432 \\
\hline Neurology (non-stroke) & 7 & 0.47 & 19 & 54 & 2.11 & 14 & $<0.001$ \\
\hline Substance abuse & 7 & 0.47 & 20 & 1 & 0.04 & 20 & 0.003 \\
\hline
\end{tabular}

* Others included a number of different causes of death, each representing a low absolute number. 
Acknowledgements We thank the Danish Pulmonary Association for supporting the study through a personal grant to the first author, and we thank Morten Pilegaard for language editing.

Contributors AF and AL: planning, statistics, writing and discussion. $\mathrm{OH}$ and $\mathrm{CW}$ : planning, writing and discussion. Rl: statistics and writing.

Funding AF was supported by a grant from the Danish Pulmonary Association.

Competing interests None declared.

Provenance and peer review Not commissioned; externally peer reviewed.

(c) Article author(s) (or their employer(s) unless otherwise stated in the text of the article) 2018. All rights reserved. No commercial use is permitted unless otherwise expressly granted.

\section{REFERENCES}

1 WHO. Global tuberculosis Report 2015. 2015 http://www.who.int/tb/publications/ global_report/en/ (accessed 18 Feb 2016).

2 European Centre for Disease Prevention and Control, WHO Regional Office for Europe. Tuberculosis surveillance and monitoring in Europe 2015, 2015.

3 Stærke NB, Hilberg 0 , Wejse C, et al. Latent tuberculosis infection is prevalent among socially marginalised citizens in Aarhus, Denmark. Dan Med J 2016;63:4-8.

4 Jensen SG, Olsen NW, Seersholm N, et al. Screening for TB by sputum culture in high-risk groups in Copenhagen, Denmark: a novel and promising approach. Thorax 2015;70:979-83.

5 Swaminathan S, Ramachandran R, Baskaran G, et al. Risk of development of tuberculosis in HIV-infected patients. Int J Tuberc Lung Dis 2000;4:839-44.

6 Bridson T, Matthiesson A, Owens L, et al. Diabetes: a Contributor to tuberculosis in Tropical Australia. Am J Trop Med Hyg 2015;93:547-8.

7 Dooley KE, Chaisson RE. Tuberculosis and diabetes mellitus: convergence of two epidemics. Lancet Infect Dis 2009;9:737-46.

8 Lönnroth K, Williams BG, Stadlin S, et al. Alcohol use as a risk factor for tuberculosis a systematic review. BMC Public Health 2008;8:289.

9 Lin HH, Ezzati M, Chang HY, et al. Association between tobacco smoking and active tuberculosis in Taiwan: prospective cohort study. Am J Respir Crit Care Med 2009; 180:475-80

10 Jayes L, Haslam PL, Gratziou CG, et al. SmokeHaz: systematic reviews and Metaanalyses of the effects of smoking on respiratory health. Chest 2016;150:164-79.

11 O'Toole RF, Shukla SD, Walters EH. TB meets COPD: an emerging global co-morbidity in human lung disease. Tuberculosis 2015;95:659-63.

12 Everatt R, Kuzmickiene I, Davidaviciene E, et al. Incidence of lung Cancer among patients with tuberculosis: a nationwide cohort study in Lithuania. Int I Tuberc Lung Dis 2016:20:757-63.

13 Nagavci BL, de Gelder R, Martikainen P, et al. Inequalities in tuberculosis mortality: long-term trends in 11 European countries. Int I Tuberc Lung Dis 2016;20:574-81.

14 Fløe A, Hilberg 0 , Wejse C, et al. The economic burden of tuberculosis in Denmark 1998-2010. cost analysis in patients and their spouses. Int I Infect Dis 2015:32:183-90.

15 Pedersen CB. The danish civil Registration System. Scand J Public Health 2011:39:22-5.

16 Deyo RA, Cherkin DC, Ciol MA. Adapting a clinical comorbidity index for use with ICD-9-CM administrative databases. J Clin Epidemiol 1992:45:613-9.

17 Sundararajan V, Henderson T, Perry C, et al. New ICD-10 version of the Charlson comorbidity index predicted in-hospital mortality. J Clin Epidemiol 2004:57:1288-94.
18 von Elm E, Altman DG, Egger M, et al. The strengthening the Reporting of Observational studies in Epidemiology (STROBE) statement: guidelines for reporting observational studies. J Clin Epidemiol 2008;61:344-9.

19 Bates MN, Khalakdina A, Pai M, et al. Risk of tuberculosis from exposure to tobacco smoke: a systematic review and meta-analysis. Arch Intern Med 2007;167:335-42.

20 O'Leary SM, Coleman MM, Chew WM, et al. Cigarette smoking impairs human pulmonary immunity to Mycobacterium tuberculosis. Am I Respir Crit Care Med 2014;190:1430-6.

21 Inghammar M, Ekbom A, Engström G, et al. COPD and the risk of tuberculosis--a population-based cohort study. PLoS One 2010;5:1-7.

22 Lee CH, Lee MC, Shu CC, et al. Risk factors for pulmonary tuberculosis in patients with chronic obstructive airway disease in Taiwan: a nationwide cohort study. BMC Infect Dis 2013;13:194

23 Shu CC, Wu HD, Yu MC, et al. Use of high-dose inhaled corticosteroids is associated with pulmonary tuberculosis in patients with chronic obstructive pulmonary disease. Medicine 2010;89:53-61.

24 Leutscher P, Madsen G, Erlandsen M, et al. Demographic and clinical characteristics in relation to patient and health system delays in a tuberculosis low-incidence country. Scand I Infect Dis 2012;44:29-36.

25 Jick SS, Lieberman ES, Rahman MU, et al. Glucocorticoid use, other associated factors, and the risk of tuberculosis. Arthritis Rheum 2006;55:19-26.

26 Sugiyama D, Nishimura K, Tamaki K, et al. Impact of smoking as a risk factor for developing rheumatoid arthritis: a meta-analysis of observational studies. Ann Rheum Dis 2010;69:70-81.

27 Jeon CY, Murray MB. Diabetes mellitus increases the risk of active tuberculosis: a systematic review of 13 observational studies. PLoS Med 2008;5:e152.

28 Kim SJ, Hong YP, Lew WJ, et al. Incidence of pulmonary tuberculosis among diabetics. Tuber Lung Dis 1995;76:529-33.

29 Andersen I, Brønnum-Hansen H, Kriegbaum M, et al. Increasing illness among people out of labor market - A Danish register-based study. Soc Sci Med 2016;156:21-8.

30 Hudolin V. Tuberculosis and alcoholism. Ann N Y Acad Sci 1975;252:353-64.

31 Pasala S, Barr T, Messaoudi I. Impact of Alcohol Abuse on the Adaptive Immune System. Alcohol Res 2015;37:185-97.

32 Mason CM, Dobard E, Zhang P, et al. Alcohol exacerbates murine pulmonary tuberculosis. Infect Immun 2004;72:2556-63.

33 Christensen AS, Roed C, Andersen $\mathrm{PH}$, et al. Long-term mortality in patients with pulmonary and extrapulmonary tuberculosis: a danish nationwide cohort study. Clin Epidemiol 2014;6:405.

34 Waitt CJ, Squire SB. A systematic review of risk factors for death in adults during and after tuberculosis treatment. Int J Tuberc Lung Dis 2011;15:871-85.

35 Surveillance in Denmark. Statens Serum Inst. 2016 http://www.ssi.dk/ Smitteberedskab/Sygdomsovervaagning.aspx (accessed 12 Jul 2016).

36 Kamper-Jørgensen Z, Andersen AB, Kok-Jensen A, et al. Clustered tuberculosis in a low-burden country: nationwide genotyping through 15 years. J Clin Microbiol 2012:50:2660-7.

37 Rock RB, Olin M, Baker CA, et al. Central nervous system tuberculosis: pathogenesis and clinical aspects. Clin Microbiol Rev 2008;21:243-61.

38 Pillaye J, Clarke A. An evaluation of completeness of tuberculosis notification in the United Kingdom. BMC Public Health 2003:3:31.

39 Baussano I, Bugiani M, Gregori D, et al. Undetected burden of tuberculosis in a lowprevalence area. Int I Tuberc Lung Dis 2006;10:415-21.

40 Leegaard A, Riis A, Kornum JB, et al. Diabetes, glycemic control, and risk of tuberculosis: a population-based case-control study. Diabetes Care 2011:34:2530-5. 